\title{
Management of Two Cases of Recurrent Anal Carcinoma
}

\author{
Eiko Klimant Maurie Markman \\ Eastern Regional Medical Center, Cancer Treatment Centers of America, \\ Philadelphia, Pa., USA
}

\section{Key Words}

Recurrent anal carcinoma $\cdot$ Irinotecan $\cdot$ Cetuximab

\begin{abstract}
Due to the low incidence of anal cancer and generally high cure rates, few second-line treatment options have been evaluated in the setting of formal clinical trials. We briefly report two cases that were both found to have wild-type K-RAS, with no epidermal growth factor receptor amplification or evidence of prior persistent human papilloma virus infection. Both cases were treated with irinotecan and cetuximab with evidence of clinical benefit in the setting of a third recurrence, as well as evidence of response to other strategies employed in their management. These cases provide support for the suggestion that treatment planning in conjunction with molecular profiling may be beneficial in such uncommon clinical settings.
\end{abstract}

(C) 2013 S. Karger AG, Basel

\section{Introduction}

Squamous cell carcinoma of the anus is a rare local-regional cancer representing approximately $2 \%$ of malignancies occurring in the gastrointestinal tract [1]. This cancer is highly responsive to standard treatment with concurrent multi-agent chemotherapy and radiation associated with only a $15 \%$ metastatic potential and a $60 \%$ cure rate [2].

However, for the subset of patients who do experience recurrence or metastatic disease, few second- or third-line treatment options exist. Due to the low incidence of this cancer and the high rate of complete or partial response to standard treatment in the primary setting, conducting clinical trials to establish the efficacy of second-line treatments has presented a difficult challenge and the only data available to inform clinical decision making in the 
treatment of these cases are largely individual or small case studies in association with alternative interventions in the face of recurrent or metastatic disease.

To date, only a few publications have reported on the response to treatment with irinotecan alone or in combination with cetuximab in patients with anal cancer who have failed standard therapy and presented with recurrence of metastatic disease.

The two patients reported here demonstrated a response to combination treatment with irinotecan and cetuximab in conjunction with the application of a molecular profiling approach. One patient was also treated with chemoradiation plus carboplatin, while the second patient demonstrated evidence of clinical benefit from the delivery of pemetrexed.

\section{Case Reports}

Case 1

A 58-year-old female presented for consultation at our center with a prior medical history of recurrent anal/rectal carcinoma first diagnosed 3 years earlier when a poorly differentiated infiltrating squamous cell carcinoma at the distal anorectal junction was found during colonoscopic evaluation performed to investigate symptoms of pain, bleeding and poor bowel function unresponsive to laxative treatment of 5 months' duration. The patient tested negative for human papilloma virus (HPV). The first recurrence 9 months later presented as an infiltrating poorly differentiated squamous cell carcinoma in the anorectal resection specimen. Lymph nodes and resection margins were negative. A biopsy of a 2-cm mass 11 months later revealed a second local recurrence of a keratinizing and nonkeratinizing anal carcinoma with basilar features. A positron emission tomography-computed tomography (PET-CT) scan of the pelvic/abdominal region revealed a new opaque mass at the anorectal junction with increased focal activity consistent with local recurrence of a primary anal carcinoma. Pathology reports at the time of the second recurrence revealed malignant metastatic squamous cell carcinoma of the anus and left retroperitoneal lymph node. The patient was staged as T2, N1 based on endoscopic ultrasound examination.

Treatment included chemoradiation therapy and concomitant cisplatin/capecitabine at the time of initial diagnosis followed by anal resection at the first recurrence. Cisplatin/capecitabine was repeated following gamma knife surgery of the anus at the time of the second recurrence.

When presenting to our center 1 year following the second recurrence, a third recurrence was suspected. A CT-guided periureteral core biopsy obtained 6 months after the initial consult confirmed the presence of a third recurrence presenting as poorly differentiated squamous cell carcinoma. The patient had additionally developed ambulatory difficulty and was dragging her left foot. Her performance status was rated as ECOG 3. An MRI revealed a small disk herniation at C6-C7 and bulging disks at L3-L4 and L4-L5 but no evidence of metastasis, and the differential diagnosis at the time was sacral plexopathy following an electromyogram study. Leg pain was eventually diagnosed as secondary to foraminal disk herniation at L3-L4 exerting compression on the exiting L3 nerve.

The patient was treated with radiation therapy for the second time (concurrent with weekly carboplatin). There was a short interval of response observed by PET scan, but after 3 months further evidence of disease progression was demonstrated.

Molecular profiling was performed on the initial pathology specimen at diagnosis. The patient expressed a wild-type genotype for K-RAS and BRAF, no epidermal growth factor receptor (EGFR) amplification by fluorescent in situ hybridization analysis, high RRMI 
Klimant et al.: Management of Two Cases of Recurrent Anal Carcinoma

expression by immunohistochemistry, low PTEN expression by immunohistochemistry, and ERCC-1 underexpression by immunohistochemistry.

Approximately 3 months after the chemoradiation with carboplatin and after discussion with the patient who wished to pursue an 'aggressive approach' to her cancer management, treatment with irinotecan plus cetuximab was initiated. The regimen was reasonably well tolerated with occasional mild diarrhea and a stable rash. Imaging studies revealed stable disease and the treatment was continued for a total of 4 cycles followed by observation. However, when there was radiographic evidence of disease progression 2 months later, the treatment program was reinitiated for several additional cycles. Of note, the time to subsequent disease progression after the documentation of a third recurrence of the anal carcinoma was 17 months. Unfortunately, with progression the patient experienced a decline in her performance status, was referred to a hospice and eventually succumbed to the disease.

Case 2

A 64-year-old female presented to our center at the time of her third recurrence with a 6 -year prior history of anal carcinoma. At this time, her ECOG score was 1 . Her primary tumor had initially been treated with concurrent chemoradiation, mitomycin $\mathrm{C}$ and 5fluorouracil (5-FU) and staged as T3NXMX. Notably, the tumor was negative for HPV. Recurrence in the right groin was noted 10 months later and managed with 7 months of cisplatin plus paclitaxel chemotherapy. She remained free of disease for approximately the next 5 years, but subsequently experienced a second recurrence in the right groin (pathology confirming poorly differentiated squamous cell carcinoma). Surgical resection was performed, with the patient experiencing a complication of deep venous thrombosis.

Unfortunately, PET-CT scan demonstrated evidence of another recurrence. Further surgery and radiation were not believed to be good options at this time, and after extensive discussions the patient was treated with the combination of irinotecan plus cetuximab. Although she experienced morbidity (diarrhea, bone marrow suppression) of the treatment and advanced disease process (cellulitis, constipation), she remained without evidence of progression of the cancer for 14 months following the initiation of irinotecan plus cetuximab.

At this point, the patient was treated with additional local radiation, maintaining a stable clinical state for approximately 11 months.

Pemetrexed was subsequently initiated, based on a molecular profiling study which revealed that the tumor exhibited low expression of thymidylate synthetase. The patient continued on this agent with stable disease for 9 months until evidence of disease progression was demonstrated.

\section{Discussion}

A recent review of survival and recurrence in an unselected national Norwegian cohort $(n=328)$ over a 7-year observational period reinforced the finding that the current standard of concomitant chemoradiotherapy (based on the outcomes of 3 clinical trials) is very effective in anal cancer management [3-6]. These investigators reported curative outcomes in $87 \%$ of patients with nonmetastatic carcinoma treated with 45.0-60.0 Gy of radiation and concomitant treatment with 5-FU and mitomycin C. Curative outcomes rose to $93 \%$ in some patients following salvage surgery. Recurrence was reported in $24 \%$ of cases with curative outcomes which were achieved in $45 \%$ of cases. Cancer-specific survival rates at 3 and 5 
Klimant et al.: Management of Two Cases of Recurrent Anal Carcinoma

years were 84 and 75\%, respectively. Additional studies have reported that the combination of 5-FU and cisplatin with radiation has a similar efficacy and may be associated with lower hematological toxicity than experienced by some patients treated with mitomycin C.

Recent efforts to improve treatment outcomes in anal squamous cell carcinoma have mainly focused on a reduction in toxicity and a prevention of local-regional failure and/or metastatic disease by exploring alternative radiation approaches or varying chemotherapeutic agents applied in the combined therapeutic approach to anal cancer. However, few alternatives exist and little focus has been applied to improving response for the subset of patients who encounter recurrence and metastatic disease and whose outcomes continue to be poor. Recurrence occurs locally most commonly, while metastatic disease usually develops by spread through lymphatic drainage to inguinal, iliac vessel and para-aortic lymph nodes [7].

The cases presented in this report involved subjects who had experienced 3 recurrences when they came to our center for evaluation having failed known 'standard therapy'. They were treated with a regimen of irinotecan plus cetuximab based on a previous report by Phan and Hoff [8].

Irinotecan is a synthetic camptothecin analog with activity against topoisomerase I and induction of $\mathrm{S}$ phase-specific cell death, and has demonstrated activity against a broad spectrum of tumors [8]. Cetuximab is a monoclonal antibody with activity against EGFR. The combination regimen may be of clinical utility even though irinotecan as a single agent may not be highly effective. This outcome is speculated to be related to molecular signaling changes that occur in the presence of both drugs. It is postulated that the inhibition of EGFR by cetuximab causes activation of the BAX pathway, caspase 8 and the NFKB pathway which render cells more susceptible to apoptosis in the context of chemotherapy [9].

These data are supported by the observation of prolonged disease stability in the two heavily pretreated anal cancer patients reported here following initiation of the combination of irinotecan plus cetuximab. This regimen has previously shown efficacy in the treatment of squamous cell cancers of the head and neck region and the cervix, which bear biological similarities to anal squamous cell carcinoma, including similar responsive rates to systemic treatment and a potential causal association with HPV. However, interestingly, our cases tested negative for HPV.

Both of our cases were also negative for EGFR amplification by fluorescent in situ hybridization. It has been described that EGFR is universally expressed in squamous cell carcinoma of the anal canal $[10,11]$. Although significant EGFR expression was noted by van Damme et al. [11] in anal cancer, neither EGFR mutation nor EGFR amplification was described, which suggests that overexpression does not result from EGFR mutation or amplification.

In a case series of patients with anal cancer, Lukan et al. [10] reported cetuximab to be efficacious and attributed this to wild-type K-RAS mutational status, which our patients also exhibited.

Van Damme et al. [11] suggested that molecular profiling in the context of anal cancer may give equivocal results based on findings that despite negative EGFR mutation and amplification in anal cancer, EGFR protein expression was detected in most cases examined in their study. They concluded that molecular screening of K-RAS and EGFR mutational status in the context of anal cancer is not an accurate predictor of efficacy of the anti-EGFR monoclonal antibody therapy. The responsiveness of our cases supports this prediction with respect to EGFR status. 
Klimant et al.: Management of Two Cases of Recurrent Anal Carcinoma

When progression after treatment with irinotecan/cetuximab was documented in case 2 , treatment with pemetrexed was initiated. Molecular profiling revealed low expression of thymidylate synthetase, suggesting a possible benefit from this antineoplastic [12].

A few other alternative treatment strategies have been tested for patients who experience multiple recurrences or metastasis of anal squamous cell carcinoma. Among the earliest regimens tested was a combination of cisplatin and 5-FU which yielded a partial response at a rate of approximately $60 \%[13,14]$. Another more aggressive regimen reporting approximately the same partial response rate with moderate toxicities included mitomycin $\mathrm{C}$, cisplatin and doxorubicin followed by bleomycin and lomustine (CCNU) in the absence of concomitant radiation therapy or surgery [15]. The median survival rate for both regimens was approximately 12 months.

Other combination regimens have been studied in only a small number of subjects and have been published as case reports. One such combination regimen for recurrent squamous cell anal cancer described recently by Golub et al. [16] included paclitaxel, ifosfamide and cisplatin (TIP regimen). Responses in this setting have also been observed with single-agent carboplatin [17], irinotecan [18], and cetuximab [10], or the combination of irinotecan and cetuximab [8].

In addition, improvements in radiation therapy approaches have been attempted to decrease damage to adjacent organs. A recent multi-institutional study by Kachnic et al. [19] reported excellent outcomes with low toxicity in the context of anal cancer treatment with dose intensity-modulated radiation therapy. Other radiation therapy approaches with promising outcomes described in the past included 3-dimensional conformational radiotherapy treatment piloted in a phase II study [20], intensity-modulated radiation therapy [21] and sequential intensity-modulated radiation therapy in a combined chemoradiological approach [22]. Improvements in radiation treatments in combination with optimization of chemotherapy may result in reduced toxicities, better outcomes and superior quality of life.

According to currently available data, EGFR expression, amplification and mutation status may not be relevant to determine treatment strategies in anal cancer. K-RAS mutations in anal cancer are very rare, and when present may predict a lack of response to treatment with cetuximab. Application of new treatment strategies in head and neck or cervical carcinomas may show efficacy in anal squamous cell carcinomas and are likely worthy of consideration in this setting. Better regimens for treatment of advanced anal cancers in second- and third-line management are needed. Advancement in molecular profiling may lead to new treatment strategies. Further research to identify new molecular targets is warranted.

\section{References}

1 Jemal A, Siegel R, Ward E, et al: Cancer statistics, 2009. CA Cancer J Clin 2009;59:225-249.

-2 Ajani JA, Winter KA, Gunderson LL, et al: Fluorouracil, mitomycin, and radiotherapy vs fluorouracil, cisplatin, and radiotherapy for carcinoma of the anal canal. JAMA 2008;299:1914-1921.

-3 UKCCCR Anal Canal Cancer Trial Working Party: Epidermoid anal cancer: results from the UKCCCR randomised trial of radiotherapy alone versus radiotherapy, 5-fluorouracil and mitomycin. Lancet 1996;348:1049-1054.

4 Bartelink H, Roelofsen F, Eschwege F, et al: Concomitant radiotherapy and chemotherapy is superior to radiotherapy alone in the treatment of locally advanced anal cancer: results of a phase III randomized trial of the European Organization for Research and Treatment of Cancer Radiotherapy and Gastrointestinal Cooperative Groups. J Clin Oncol 1997;15:2040-2049.

-5 Flam M, John M, Pajak TF, et al: Role of mitomycin in combination with fluorouracil and radiotherapy, and of salvage chemoradiation in the definitive nonsurgical treatment of epidermoid carcinoma of the anal canal: results of a phase III randomized intergroup study. J Clin Oncol 1996;14:2527-2539. 


\section{Case Reports in Oncology}

\begin{tabular}{l|l}
\hline Case Rep Oncol 2013;6:456-461 \\
\hline DOI: $10.1159 / 000355004$ & $\begin{array}{l}\text { C 2013 S. Karger AG, Basel } \\
\text { www.karger.com/cro }\end{array}$ \\
\hline
\end{tabular}

Klimant et al.: Management of Two Cases of Recurrent Anal Carcinoma

6 Bentzen AG, Guren MG, Wanderas EH, et al: Chemoradiotherapy of anal carcinoma: survival and recurrence in an unselected national cohort. Int J Radiat Oncol Biol Phys 2012;83:e173-e180.

7 Renehan A, Saunders MP, Hill J, et al: Radiotherapy versus combined modality therapy for anal carcinoma. Cochrane Database Syst Rev 2008;1:CD004652.

8 Phan LK, Hoff PM: Evidence of clinical activity for cetuximab combined with irinotecan in a patient with refractory anal canal squamous-cell carcinoma: report of a case. Dis Colon Rectum 2006;50:395-398.

-9 Xu Y, Villalona-Calero MA: Irinotecan: mechanisms of tumor resistance and novel strategies for modulating its activity. Ann Oncol 2002;13:1841-1851.

10 Lukan N, Strobel P, Willer A, et al: Cetuximab-based treatment of metastatic anal cancer: correlation of response with KRAS mutational status. Oncology 2009;77:293-299.

11 Van Damme N, Deron P, Van Roy N, et al: Epidermal growth factor receptor and KRAS status in two cohorts of squamous cell carcinomas. BMC Cancer 2010;10:189.

12 Adjei AA: Pemetrexed (Alimta): a novel multitargeted antineoplastic agent. Clin Cancer Res 2004;10:4276s4280s.

13 Jaiyesimi IA, Pazdur R: Cisplatin and 5-fluorouracil as salvage therapy for recurrent metastatic squamous cell carcinoma of the anal canal. Am J Clin Oncol 1993;16:536-540.

14 Hung A, Crane C, Delclos M, et al: Cisplatin-based combined modality therapy for anal carcinoma: a wider therapeutic index. Cancer 2003;97:1195-1202.

15 Jhawer M, Mani S, Lefkopoulou M, et al: Phase II study of mitomycin-C, adriamycin, cisplatin (MAP) and bleomycin-CCNU in patients with advanced cancer of the anal canal: an Eastern Cooperative Oncology Group study E7282. Invest New Drugs 2006;24:447-454.

16 Golub DV, Cahid Civelek A, Sharma VR: A regimen of taxol, ifosfamide, and platinum for recurrent advanced squamous cell carcinoma of the anal canal. Chemother Res Pract 2011;2011:163736.

17 Evans TR, Mansi JL, Glees JP: Response of metastatic anal carcinoma to single agent carboplatin. Clin Oncol (R Coll Radiol) 1993;5:57-58.

18 Grifalchi F, Padovani A, Romeo F, et al: Response of metastatic epidemoid anal cancer to single agent irinotecan: a case report. Tumori 2001;87:58-59.

19 Kachnic LA, Tsai HK, Coen JJ, et al: Dose-painted intensity-modulated radiation therapy for anal cancer: a multi-institutional report of acute toxicity and response to therapy. Int J Radiat Oncol Biol Phys 2012;82:153-158.

20 Vuong T, Devic S, Belliveau P, et al: Contribution of conformal therapy in the treatment of anal canal carcinoma with combined chemotherapy and radiotherapy: results of a phase II study. J Radiat Oncol Biol Phys 2003;56:823-831.

-21 Czito BG, Willett CG: Current management of anal canal cancer. Curr Oncol Reports 2009;11:186-192.

-22 Salama JK, Mell LK, Schomas DA, et al: Concurrent chemotherapy and intensity-modulated radiation therapy for anal canal cancer patients: a multicenter experience. J Clin Oncol 2007;25:4581-4586. 